\title{
EFEITOS DO EXERCÍCIO FÍSICO NO TRATAMENTO DA ARTRITE IDIOPÁTICA JUVENIL: UMA REVISÃO SISTEMÁTICA
}

\author{
EFFECTS OF PHISICAL EXERCISE IN TREATMENT OF JUVENILE IDIOPATHIC ARTHRITIS: \\ A SISTEMATIC REVIEW
}

\author{
Josiane Santos da Silveira ${ }^{a}$, Vágner Winck Moura ${ }^{b}$, Adriana Marques Toigo ${ }^{c}$ \\ ajosi.santeira@hotmail.com, bvagner.winck@gmail.com, cadrytoigo@terra.com.br \\ Centro Universitário La Salle - Canoas (RS), Brasil
}

Data de recebimento do artigo: 13/07/2017

Data de aceite do artigo: 13/08/2017

\section{RESUMO}

Introdução: A Artrite Idiopática Juvenil (AIJ) é uma doença autoimune, crônica, de etiologia desconhecida, que acomete crianças menores de 16 anos de idade, caracterizada por dor articular e edema, podendo progredir para comprometimento da cartilagem e do osso, resultando em perda da funçáo e deformidades articulares. Exercícios físicos têm como finalidade a melhoria do desempenho físico, logo, pode-se pensar neles como terapia adjuvante ao tratamento convencional da AIJ. Contudo, questiona-se qual seria o tipo mais apropriado para os portadores da doença. Objetivo: Buscar evidências na literatura científica sobre os potenciais benefícios do exercício físico na AIJ. Método: Busca nas bases de dados PubMed e SciELO, utilizando-se os termos artrite reumatoide, artrite reumatoide juvenil, artrite idiopática juvenil, atividade fisica, exercício fisico, treinamento físico, ginástica, corrida e esporte. Resultados: Sete estudos foram selecionados para análise dos resultados, pois enquadravam-se nos critérios de inclusão. Os poucos estudos sobre os efeitos do exercício físico na AIJ se diferenciam bastante nos testes e protocolos de treinamento, tornando difícil o estabelecimento de comparaçôes. Conclusáo: Embora o conjunto dos fatores que contribuem para a ocorrência da artrite reumatoide (AR) seja extensamente estudado, principalmente fatores genéticos e imunológicos, os mecanismos precisos envolvidos na iniciação, progressão e destruição autoimune presentes na AIJ não estão totalmente elucidados. As evidências explicitadas sugerem que o exercício pode e deve ser estimulado e incorporado à terapia medicamentosa para beneficiar indivíduos com AIJ, atenuando sintomas clínicos relacionados à doença. Não foram observados efeitos prejudiciais relacionados ao exercício, corroborando este como terapia adjuvante ao tratamento da AIJ.

Palavras-chave: Artrite juvenil; exercício; benefícios.

\section{ABSTRACT}

Introduction: The Juvenile Idiopathic Arthritis (JIA) is an autoimmune disease, chronic, of unknown etiology, that affects children under 16 years of age, characterized by joint pain and swelling that may progress to impairment of cartilage and bone, resulting in loss of function and joint deformities. Physical exercises are supposed to improve physical performance, so it can be suggested as adjunctive therapy to conventional treatment of JIA. However, the question is what would be the most appropriate exercise for these individuals. Objective: To look for evidence in the scientific literature about the potential benefits of the practice of physical exercise by people with JIA. Method: A search in PubMed and SciELO databases, using the terms rheumatoid arthritis, juvenile rheumatoid arthritis, juvenile idiopathic arthritis, physical activity, physical exercise, physical training, gymnastics, running and sport was held. Results: Seven papers were selected for analysis of the results, as they fulfilled the inclusion criteria. As there were few studies on the effects of exercise in JIA and the authors have used different tests and training protocols, it was difficult to establish some comparisons. Conclusion: Although all the factors that contribute to the occurrence of rheumatoid arthritis (RA) are widely studied, mainly genetic and immunological factors, the precise mechanisms involved in the initiation, progression and autoimmune destruction present in JIA are not fully elucidated. Evidence specified suggests that 
exercise can and should be encouraged and added to the drug treatment to benefit people with JIA, in order to reduce clinical symptoms related to the disease. There were no adverse effects related to exercise, confirming this as adjunctive therapy to the treatment of JIA.

Keywords: Juvenile arthritis; exercise; benefits.

\section{Introdução}

A inatividade física (IF) aumenta substancialmente a incidência relativa de doenças cerebrovasculares, doenças isquêmicas do coração, hipertensão, diabetes e osteoporose ${ }^{1}$. A IF é considerada também uma das principais responsáveis pelo aumento da incidência de obesidade infantil, doenças cardiovasculares, diabetes e problemas psicológicos ${ }^{2}$, além de representar um elemento agravante do estado geral de saúde em crianças e adolescentes acometidos por várias doenças, incluindo as neuromusculares e osteoarticulares ${ }^{3}$.

As doenças inflamatórias constituem um grupo complexo e heterogêneo de doenças, sendo causa importante de morbidade e mortalidade ${ }^{4}$. Já as doenças autoimunes são caracterizadas por desordens resultantes do sistema imunitário, que ataca os próprios tecidos do corpo e provoca também um aumento da inflamaçáo 5 . Qualquer distúrbio que resulte em perda de função ou destruição de tecido normal desencadeia respostas imunes humorais e celulares para os próprios constituintes de tecidos do corpo ${ }^{6}$.

A artrite reumatoide (AR) é uma doença autoimune sistêmica do tecido conjuntivo, que acomete predominantemente as articulaçóes diartrodiais e as estruturas periarticulares, tendo como principal característica o processo inflamatório de caráter crônico ${ }^{7}$. A causa da $\mathrm{AR}$ não é conhecida e o foco da inflamação é o tecido sinovial, o que reveste as articulaçóes ${ }^{8}$. O resultado desse processo autoimune é a inflamação das articulaçôes e consequente dor, inchaço e rubor na regiáo acometida, o que ocorre principalmente nas mãos e nos pés, levando à deformidade e à destruição das articulaçóes por erosão da cartilagem articular, isto é, da membrana sinovial, chegando até os ossos ${ }^{9}$. Por ser sistêmica, embora a principal característica da AR seja a inflamaçáo das articulaçôes, várias regiōes do corpo também podem ser comprometidas ${ }^{10}$.

A AR afeta entre $0,5 \%$ e $1 \%$ da população mundial adulta e cerca de três vezes mais mulheres do que homens; indivíduos com histórico familiar de AR têm maior probabilidade de desenvolver a doença pela predisposição genética. A artrite reumatoide juvenil, também conhecida como artrite idiopática juvenil (AIJ), é uma doença autoimune, crônica, de etiologia desconheci$\mathrm{da}$, que acomete crianças menores de 16 anos de idade, caracterizada por dor articular e edema, que pode progredir para comprometimento da cartilagem e do osso, resultando em perda da funçáo e deformidades articulares. A incidência da AIJ é desconhecida no Brasil, embora seja considerada uma das doenças crônicas mais comuns da infância, com uma prevalência de cerca de 1:1000 crianças, conforme dados provenientes de países da América do Norte e Europa ${ }^{10,11}$. A AIJ frequentemente persiste na idade adulta e pode resultar em significativa morbidade a longo prazo, incluindo deficiência física ${ }^{7,11,12}$.

\section{Diagnóstico}

A etiologia precisa que desencadeia o processo autoimune e os mecanismos patogênicos iniciais não estão totalmente esclarecidos, podendo incluir infecçóes, traumas, alteraçóes imunológicas e estresse ${ }^{13}$.

O diagnóstico da AIJ é amplo ${ }^{14}$ e realizado basicamente de forma clínica, com base nos padróes propostos pela International League of Associations for Rheumatology (ILAR), acometendo crianças com idade inferior a 16 anos de idade, com artrite em uma ou mais articulaçôes, duração de artrite em uma mesma articulação por no mínimo 6 semanas ${ }^{15,16}$ e só pode ser confirmado após da exclusão de outras patologias, tais como infecçóes, câncer infantil, doenças ósseas, lúpus, entre outras ${ }^{17}$. Em estágios iniciais, algumas crianças podem não apresentar quadro de dor ou inchaço perceptível na articulação acometida, dificultando ainda mais o trabalho dos médicos ${ }^{10}$.

Não há um único teste ou exame que confirme a AIJ; em vez disso, o médico faz o diagnóstico analisando os sintomas e resultados dos exames físicos, laboratoriais e de raios- $\mathrm{X}^{8}$. Exames de sangue podem auxiliar a confirmar o diagnóstico da doença. Os principais indicativos incluem a anemia (baixa contagem de células vermelhas do sangue) e o fator reumatoide (um anticorpo, ou uma proteína do sangue, encontrada em cerca de $80 \%$ dos pacientes com AR, todavia encontrada em apenas 30\% dos indivíduos em estado inicial da doença). Raios-X também podem auxiliar no diagnóstico, contudo não são precisos nos estágios iniciais da doença, embora possam ser úteis para fins de acompanhamento de sua evolução. Muitas vezes, ressonância magnética e ultrassonografia são feitas para ajudar a avaliar a gravidade da doença ${ }^{8}$. 


\section{Sintomatologia}

A AIJ se inicia com sintomas sutis, tais como febre alta diária (acima de $39^{\circ} \mathrm{C}$ por períodos maiores do que 2 semanas), fraqueza, anorexia, irritabilidade, regressão psicológica, atitude protetora com alguma articulação, recusa para engatinhar ou caminhar. Com a evolução da doença, podem ser verificados temperatura alta, inchaço e dor acentuada à palpação e movimentação, certa dificuldade na movimentação ao acordar (rigidez matinal) ou após algum tempo de inatividade, espasmos, dificuldade ao desenvolver atividades motoras finas, diminuição da mobilidade nas articulaçôes afetadas, crepitação e atrofia muscular ${ }^{7,10}$.

\section{Critérios de classificação}

A AIJ pode ser subdividida em sistêmica (caracterizada por febre intermitente, com duração de, no mínimo, 15 dias, associada a erupção cutânea, raramente pruriginosa e localizada preferencialmente no tronco e na porção proximal de membros), oligoarticular (acometendo de uma a quatro articulaçóes), poliarticular (comprometendo cinco ou mais articulaçóes, com fator reumatoide positivo ou não), artrite relacionada à entesite (podendo apresentar-se como oligoartrite ou poliartrite, com comprometimento assimétrico das articulaçóes dos membros inferiores e do esqueleto axial, incluindo a espondilite anquilosante juvenil, a artrite relacionada ao antígeno HLA-B27 e a artrite relacionada à doença inflamatória intestinal), artrite psoriásica (diagnosticada quando há artrite na presença de psoríase, história familiar de psoríase, dactilite e alteraçôes nas unhas aparentemente em forma de dedal) ${ }^{14,15}$.

\section{Fisiopatologia e tratamento}

O tratamento para a AIJ assemelha-se ao das outras categorias de doenças inflamatórias, quer sejam de caráter crônico ou não, e inclui tanto medidas farmacológicas quanto não farmacológicas, tais como: fisioterapia, exercícios físicos, cuidados alimentares, entre outros ${ }^{18,19}$.

A AIJ pode causar rigidez matinal ou rigidez após um determinado tempo de inatividade física, que pode ser uma boa forma de monitoramento da progressáo da doença. Quanto maior o período de rigidez, maior o comprometimento articular. Algumas medidas iniciais para aliviar a rigidez podem ser adotadas, tais como tomar um banho quente, usar uma bolsa com gelo na região afetada, ou ainda realizar exercícios de alongamento ${ }^{13,15}$.
O tratamento deve ser contínuo e seus principais objetivos centram-se em controle da inflamaçáo, diminuição da dor, prevenção de dano irreversível nas articulações, melhora da função articular, prevenção de lesôes articulares, controle da atividade e busca pela regressão da doença, prevenindo uma série de sucessivas outras doenças, tais como distúrbios de crescimento, lesōes articulares e limitaçóes funcionais, buscando subsidiar uma melhor qualidade de vida e desenvolvimento dentro dos padróes da normalidade ${ }^{18,19}$.

\section{Farmacologia}

A medicação é comumente utilizada de forma isolada ou em associaçôes, levando em consideração a individualidade biológica, o estágio da doença e a especificidade de cada indivíduo ${ }^{7,10}$.

Os medicamentos comumente utilizados no tratamento da AIJ são:

1) anti-inflamatórios não esteroides (AINES): utilizados para controle da dor e da atividade inflamatória em curto prazo. Sua toxidade pode ser verificada por meio de exames laboratoriais, permitindo o seu uso seguro. A via de administração pode ser oral ou em comprimido ${ }^{10}$.

2) corticosteroides: são os medicamentos anti-inflamatórios mais potentes e são normalmente utilizados para tratamento quando a doença não responde a outros medicamentos. Devido a sua alta toxicidade e por apresentarem muitos efeitos colaterais, são prescritos com cautela e o tratamento não deve ser de longo prazo ${ }^{10}$.

3) antirreumáticos modificadores da doença (Disease Modifying Anti-rheumatoid Drugs - DMARD): também conhecidos como anti-inflamatórios de ação lenta, não são destinados ao alívio da dor ou à redução do quadro inflamatório imediato; seus benefícios podem ser notados em longo prazo, modificando a evolução natural da doença articular. Podem ser associados a outros AINES. Por serem medicamentos mais fortes, faz-se necessária a realização frequente de exames laboratoriais para monitorar os níveis de toxicidade ${ }^{10}$.

4) analgésicos: são destinados ao alivio da dor e não aliviam a inflamação, todavia são prescritos em associação a outros medicamentos ${ }^{10}$.

5) medicamentos biológicos: os medicamentos biológicos compóem uma nova classe de medicamentos, feitos de proteínas sintéticas, utilizadas com a finalidade de diminuir ou interromper a resposta imunológica prejudicial que leva à inflamação das articulaçôes ${ }^{10}$. 


\section{Intervenção cirúrgica}

Em um primeiro momento, mesmo se há evidência de deformidade articular, podem ser utilizadas talas gessadas para manter ou retornar a articulaçáo doente a posição normal e aliviar a dor. As talas são normalmente feitas por um fisioterapeuta ${ }^{7,9}$.

A intervenção cirúrgica é indicada para restauração das funçóes motoras, diminuição da dor e correçáo de deformidades articulares; algumas são indicadas somente em casos de dor sem controle e limitaçóes funcionais incapacitantes ${ }^{7}$.

Os tratamentos cirúrgicos mais utilizados são para a correção e liberação de partes moles com contraturas fixas; alívio de compressão nervosa; correção do quadril (artroplastia); correção de deformidades em dedos e artelhos; prolongamento da vida útil da cartilagem (sinovectomias); realinhamento ósseo (osteotomia) e fusão da placa de crescimento (epifisiodese) ${ }^{9,16}$.

\section{Exercícios físicos}

A atividade física tem como finalidade a melhoria do desempenho corporal por meio da sistematização e organização de exercícios físicos ${ }^{20}$. Dessa forma, pode-se pensar na atividade e no exercício físicos como terapias adjuvantes ao tratamento convencional da AIJ. Os efeitos terapêuticos dos exercícios físicos em doenças reumatológicas pediátricas têm sido alvo de investigação. Em conjunto, algumas publicaçóes têm revelado grande potencial terapêutico do exercício físico para indivíduos com AIJ, além daqueles com lúpus eritematoso sistêmico juvenil (LESJ), dermatomiosite juvenil (DMJ) e fibromialgia juvenil (FMJ) $3,21,22$.

Contudo, é escasso o número de estudos controlados randomizados e com amostragens significativas, com intervenção por meio da atividade física ou do exercício físico. Questiona-se, ainda, se há algum tipo de exercício físico mais apropriado para a população com AIJ a fim de lhe proporcionar benefícios físicos e fisiológicos. Logo, o objetivo desta breve revisão foi discutir os potenciais efeitos dos principais achados científicos sobre o exercício físico na AIJ.

\section{Metodologia}

O presente estudo configurou-se como uma revisão sistemática da literatura cujas bases de dados consultadas foram SciELO e PubMed. Os unitermos de busca independentes e associados foram: artrite reumatoide, artrite reumatoide juvenil, artrite idiopática juvenil, atividade fisica, exercício fisico, treinamento físico, ginástica, corrida e esporte; rheumatoid arthritis, juvenile rheumatoid arthritis, juvenile idiopathic arthritis, physical activity, physical exercise, physical training, gymnastics, running e sport. O cruzamento dos termos supracitados, através do operador boleano "AND", também foi executado. O período de pesquisa definido para a revisão foi limitado a 10 anos, até junho de 2016, e incluiu estudos publicados nas línguas portuguesa e inglesa. Todos os cruzamentos efetuados estấo diagramados na Tabela 1:

Tabela 1: Termos e números de artigos localizados nas respectivas bases de dados

\begin{tabular}{|c|c|c|c|}
\hline \multicolumn{4}{|c|}{1 - rheumatoid arthritis } \\
\hline \multicolumn{4}{|c|}{2 - juvenile rheumatoid arthritis } \\
\hline \multicolumn{4}{|c|}{3 - juvenile idiopathic arthritis } \\
\hline \multicolumn{4}{|c|}{ 4-physical activity } \\
\hline \multicolumn{4}{|c|}{ 5-physical exercise } \\
\hline \multicolumn{4}{|c|}{$6-$ physical training } \\
\hline \multicolumn{4}{|c|}{ 7-gymnastics } \\
\hline \multicolumn{4}{|c|}{8 - running } \\
\hline \multicolumn{4}{|c|}{$9-$ sports } \\
\hline \multirow{2}{*}{$\begin{array}{l}\text { SciELO } \\
\text { Termos }\end{array}$} & \multicolumn{3}{|c|}{ PubMed } \\
\hline & $\begin{array}{l}\text { Número de } \\
\text { artigos }\end{array}$ & Termos & $\begin{array}{l}\text { Número de } \\
\text { artigos }\end{array}$ \\
\hline 1 & 330 & 1 & 11 \\
\hline 2 & 24 & 2 & 9 \\
\hline 3 & 46 & 3 & 13 \\
\hline 4 & 2343 & 4 & 180 \\
\hline 5 & 680 & 5 & 18492 \\
\hline 6 & 340 & 6 & 21442 \\
\hline 7 & 170 & 7 & 11921 \\
\hline 8 & 380 & 8 & 1562 \\
\hline 9 & 246 & 9 & 11095 \\
\hline 2 AND 4 & 1 & 2 AND 4 & 15 \\
\hline 2 AND 5 & 1 & 2 AND 5 & 12 \\
\hline 2 AND 6 & 1 & 2 AND 6 & 9 \\
\hline 2 AND 7 & 0 & 2 AND 7 & 0 \\
\hline 2 AND 8 & 0 & 2 AND 8 & 0 \\
\hline 2 AND 9 & 0 & 2 AND 9 & 0 \\
\hline 3 AND 4 & 1 & 3 AND 4 & 15 \\
\hline 3 AND 5 & 2 & 3 AND 5 & 12 \\
\hline 3 AND 6 & 1 & 3 AND 6 & 9 \\
\hline 3 AND 7 & 0 & 3 AND 7 & 0 \\
\hline 3 AND 8 & 0 & 3 AND 8 & 0 \\
\hline 3 AND 9 & 0 & 3 AND 9 & 3 \\
\hline
\end{tabular}

Fonte: Elaboração própria, 2017.

Os critérios de inclusão foram: artigos com pessoas vivendo com artrite idiopática juvenil; com intervenção por meio da atividade física, exercício físico ou treinamento físico; e artigos publicados nos últimos 10 
anos. Os critérios de exclusão foram: artigos de revisão e artigos com utilização de modelos experimentais. Os artigos localizados foram avaliados obedecendo aos critérios de inclusáo e exclusão a fim de aperfeiçoar o processo de seleçáo, uma vez que são escassos os estudos controlados randomizados e com amostragens significativas, com intervenção por meio da atividade física ou do treinamento físico para indivíduos acometidas por AIJ.

\section{Resultados}

Após a busca nos bancos de dados, foi obtido um total de 7 trabalhos que se enquadraram nos critérios de inclusão, conforme ilustra o organograma apresentado na Figura 1.
Figura 1: Organograma dos artigos incluídos

18 artigos encontrados nas bases de dados, com a junção dos termos artrite idiopática juvenil + atividade física/exercício físico/ treinamento físico/esportes

7 artigos selecionados conforme critérios de inclusão

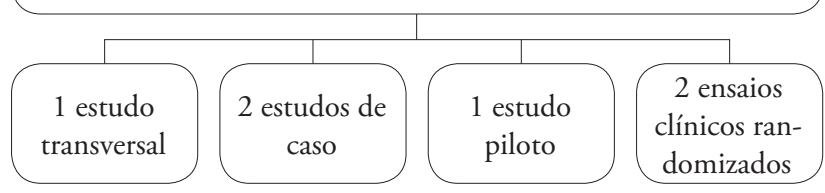

Fonte: Elaboração própria, 2017.

A Tabela 2 apresenta, em ordem cronológica, um resumo dos estudos encontrados na literatura científica.

Tabela 2: Descrição dos artigos selecionados

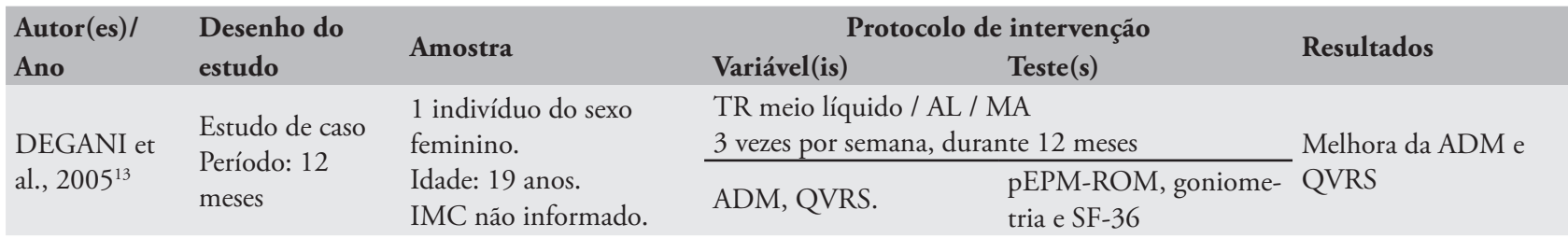

Programa de exercícios em circuito envolvendo

TR em meio líquido.

Estudo piloto para um maior

SINGH- estudo do tipo

GREWAL et randomizado

al., $2006^{23}$

controlado cego

Período: 12

semanas

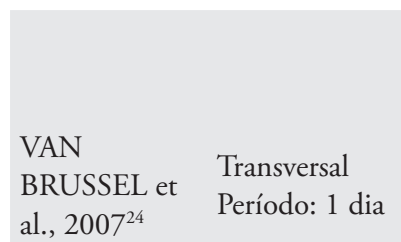

Indivíduos de ambos os

sexos.

GE ( $\mathrm{n}=62)$; AIJ.

Idade: $11,9 \pm 2,2$ anos. CA, CAN, CF, percen-

$\mathrm{IMC}=18,7 \pm 3,7 \mathrm{~kg} / \mathrm{m}^{2}$. tual de gordura, status

$\mathrm{GC}(\mathrm{n}=$ não informa- articular.

do); saudáveis.

Idade: $12,3 \pm 2,5$ anos.

IMC $=18,0 \pm 2,6 \mathrm{~kg} / \mathrm{m}^{2}$

Programa de exercícios (TR/TA) em bicicleta

ergométrica, esteira e Fitball.

2 vezes por semana durante 12 semanas.

Dor, PE de acordo com

FC alcançada, EM.

Adolescentes de ambos

os sexos.

GE (n = 25); AIJ.

LELIEVELD Transversal Idade: $17,1 \pm 0,7$ anos.

et al., $2007^{25} \quad$ Período $=1 \mathrm{dia}$
CA, CAN, CF. $\quad$ C-HAQ.
$\mathrm{IMC}=20,8 \pm 2,7 \mathrm{~kg} / \mathrm{m}^{2}$

GC ( $\mathrm{n}=27)$; saudáveis.

Idade: 17,0 \pm 0,6 anos.

$\mathrm{IMC}=21,1 \pm 2,3 \mathrm{~kg} / \mathrm{m}^{2}$
Redução do custo energético de locomoção; aumento do $\mathrm{VO}_{2 \text { pico; }}$ aumento da potência anaeróbia de perna pico de potência anaeróbia de braço foram reduzidos de forma significativa WAnT, C-HAQ, teste Portadores de AIJ tem cineantropométrico menor capacidade de 7 dobras cutâneas aeróbia e anaeróbia que (protocolo de Pollack) e seus pares saudáveis
Portadores de AIJ tem menor capacidade aeróbia e anaeróbia que seus pares saudáveis
SLBET, WAnT e

\section{Juvenile Arthritis Funcitional Status e escala de Borg. \\ Ergoespirometria, pEPM-ROM \\ Teste de consumo nio, teste de $\mathrm{VO}_{2}$ pico, e

, teste
rico
âneas
ollack) e


Tabela 2: Continuação.

\begin{tabular}{|c|c|c|c|c|c|}
\hline \multirow{2}{*}{$\begin{array}{l}\text { Autor(es)/ } \\
\text { Ano }\end{array}$} & \multirow{2}{*}{$\begin{array}{l}\text { Desenho do } \\
\text { estudo }\end{array}$} & \multirow{2}{*}{ Amostra } & \multicolumn{2}{|c|}{ Protocolo de intervenção } & \multirow{2}{*}{ Resultados } \\
\hline & & & Variável(is) & Teste(s) & \\
\hline & & & \multicolumn{2}{|c|}{$\begin{array}{l}\text { TR meio líquido / AL / MA e atividades lúdicas } \\
2 \text { vezes por semana, durante } 5 \text { semanas }\end{array}$} & \multirow[b]{2}{*}{$\begin{array}{l}\text { Melhora na ADM, fle- } \\
\text { xibilidade, capacidade } \\
\text { funcional, estado geral } \\
\text { de saúde e vitalidade, } \\
\text { redução da dor e de va- } \\
\text { lores de cirtometria em } \\
\text { membros inferiores. }\end{array}$} \\
\hline $\begin{array}{l}\text { SANTONI et } \\
\text { al., } 2007^{12}\end{array}$ & $\begin{array}{l}\text { Estudo de caso } \\
\text { Período: } 5 \\
\text { semanas }\end{array}$ & $\begin{array}{l}1 \text { indivíduo do sexo } \\
\text { masculino. } \\
\text { Idade: } 5 \text { anos. } \\
\text { IMC não informado. }\end{array}$ & CF, ADM, QVRS. & $\begin{array}{l}\text { Testes de amplitude de } \\
\text { movimento articular, } \\
\text { força muscular, flexi- } \\
\text { bilidade, cirtometria } \\
\text { de membros inferiores, } \\
\text { anamnese, inspeção, } \\
\text { palpação, avaliação da } \\
\text { marcha e avaliaçáo pos- } \\
\text { tural (não especificados) } \\
\text { e SF-36. }\end{array}$ & \\
\hline \multirow[t]{2}{*}{$\begin{array}{l}\text { SINGH- } \\
\text { GREWAL et } \\
\text { al., } 2007^{26}\end{array}$} & \multirow[t]{2}{*}{$\begin{array}{l}\text { Randomizado } \\
\text { Controlado } \\
\text { Cego } \\
\text { Período: } 12 \\
\text { semanas }\end{array}$} & \multirow{2}{*}{$\begin{array}{l}\text { Indivíduos de ambos os } \\
\text { sexos com AIJ. } \\
\text { GE ( } \mathrm{n}=41) \\
\text { Idade: } 11,7 \pm 2,5 \text { anos } \\
\text { GC ( } \mathrm{n}=39) \\
\text { Idade: } 11,5 \pm 2,4 \text { anos }\end{array}$} & \multicolumn{2}{|c|}{$\begin{array}{l}\text { GE: } 10 \text { ' de aquecimento e AL, } 30 \text { ' TA com dança } \\
\text { e cardio-karate com intensidades aumentando } \\
\text { progressivamente de leve a moderada a intensa e } \\
5 \text { ' AL ativo. } \\
\text { GC: } 18 \text { exercícios de qigong (semelhantes ao } \\
\text { tai chi) prescritos de forma a evitar adaptaçôes } \\
\text { aeróbicas. } \\
3 \text { vezes por semana, durante } 12 \text { semanas. }\end{array}$} & \multirow{2}{*}{$\begin{array}{l}\text { Melhora nas funções } \\
\text { físicas. } \\
\text { A intensidade do TA } \\
\text { não provocou benefí- } \\
\text { cios adicionais. } \\
\text { Programas de intensi- } \\
\text { dade mais baixa como } \\
\text { o qigong são de maior } \\
\text { aderência e promovem } \\
\text { benefícios equivalen- } \\
\text { tes a programas de } \\
\text { exercícios de maior } \\
\text { intensidade. }\end{array}$} \\
\hline & & & $\mathrm{CA}, \mathrm{CAN}, \mathrm{CF}$ & $\begin{array}{l}\text { Teste de } \mathrm{VO}_{2} S_{\text {ubmax }} \text {, } \\
\text { Teste de } \mathrm{VO}_{2 \text { máx }} \\
\text { WAnT, C-HAQ }\end{array}$ & \\
\hline \multirow[t]{2}{*}{$\begin{array}{l}\text { TARAKCI et } \\
\text { al., } 2012^{27}\end{array}$} & \multirow[t]{2}{*}{$\begin{array}{l}\text { Randomizado, } \\
\text { controlado, cego } \\
\text { Período: } 12 \\
\text { semanas }\end{array}$} & \multirow{2}{*}{$\begin{array}{l}\text { Indivíduos de ambos os } \\
\text { sexos com AIJ. } \\
\text { GE }(\mathrm{n}=43) . \\
\text { Idade: } 10,02 \pm 3,44 \\
\text { anos. } \\
\mathrm{IMC}=17,28 \pm 3,52 \mathrm{~kg} / \\
\mathrm{m}^{2} . \\
\text { GC ( } \mathrm{n}=38) \\
\text { Idade: } 10,82 \pm 4 \text { anos. } \\
\mathrm{IMC}=18,22 \pm 3,60 \\
\mathrm{~kg} / \mathrm{m}^{2}\end{array}$} & \multicolumn{2}{|c|}{$\begin{array}{l}\text { GC = não realizou nenhum exercício. } \\
\text { GE = realizou um programa de exercícios domés- } \\
\text { ticos incluindo aquecimento, TR com Theraband, } \\
\text { AL, EP, EF } \\
1 \text { vez por semana com supervisão do fisioterapeuta } \\
\text { no hospital e } 3 \text { vezes por semana com supervisão } \\
\text { dos pais em casa. }\end{array}$} & \multirow{2}{*}{$\begin{array}{l}\text { Melhora na capacidade } \\
\text { funcional, mobilidade } \\
\text { articular, fortalecimen- } \\
\text { to muscular, flexibilida- } \\
\text { de, postura, qualidade } \\
\text { de vida e redução da } \\
\text { dor. }\end{array}$} \\
\hline & & & CF, dor, QVRS. & $\begin{array}{l}\text { Teste de caminhada de } \\
6 \text { ', escala visual analógi- } \\
\text { ca, C-HAQ e PedsQL }\end{array}$ & \\
\hline
\end{tabular}

Nota: GE = grupo experimental; GC = grupo controle; $\mathrm{AIJ}=$ artrite idiopática juvenil; $\mathrm{CA}$ = capacidade aeróbica; $\mathrm{CAN}=$ capacidade anaeróbica; $\mathrm{CF}=$ capacidade funcional; $\mathrm{TA}=$ treinamento aeróbio; $\mathrm{TR}=$ treinamento resistido; $\mathrm{MA}=$ mobilização articular; $\mathrm{AL}=$ alongamentos; $\mathrm{ADM}=$ amplitude de movimento articular; QVRS = qualidade de vida relacionada a saúde; $\mathrm{EP}=$ exercícios posturais; $\mathrm{EF}$ = exercícios funcionais; $\mathrm{PE}$ = percepção de esforço; $\mathrm{EM}=$ eficiência metabólica; kg/m2 = quilograma por metro quadrado; C-HAQ: Childhood Health Assessment Questionnaire; SF-36 = Medical Outcomes Study Short-form 36; pEPM-ROM = Escala de Amplitude de Movimento da Escola Paulista de Medicina Pediátrica; WAnT = Wingate Anaerobic Exercise Test; SLBET = Symptom Limited Bicycle Ergometry Test; PedsQL = Pediatric Quality of Life Inventory Module Arthritis; VO2máx = consumo máximo de oxigênio; VO2submax = consumo submáximo de oxigênio; VO2pico = pico de consumo de oxigênio.

Fonte: Elaboração própria, 2017.

Degani et al. ${ }^{13}$, em um estudo com uma voluntária de 19 anos de idade, portadora de AIJ do tipo sistêmica, submetida a um programa de fisioterapia aquática, buscaram evidenciar uma relação entre a melhora na qualidade de vida de portadores de AIJ e a prática de exercícios. Foram avaliadas a amplitude de movimento articular (ADM), por goniometria e pelo escore da Escala de Amplitude de Movimento da Escola Paulista de Medicina Pediátrica (pEPM-ROM), e a qualidade de vida relacionada à saúde (QVRS), com o questionário Medical Outcomes Study Short-form 36 (SF-36). Foram realizadas 3 sessóes semanais de 45 a $60 \mathrm{mi}-$ nutos cada, por 12 meses, sendo essa a única terapia utilizada além do tratamento medicamentoso, o qual não sofreu alteraçóes nesse período. Foram realizados exercícios planares e em diagonal, alongamentos ativos 
e passivos, mobilizaçóes passivas, exercícios respiratórios, hidrodinâmicos e de flutuabilidade, treino de marcha, reeducação postural, exercícios aeróbios, natação adaptada e técnicas do conceito Halliwick, do método dos anéis de Bad Ragaz, do Aquastretshing e do Watsu. Antes da intervenção, a voluntária dependia de muletas para deslocar-se por trajetos de até 500 metros; após a terapia, realizava marcha independente por até 1000 metros. Melhoras na ADM foram observadas bilateralmente na maioria das articulaçôes, contudo a avaliação da QVRS apresentou níveis muito baixos. Já na avaliação final pôde ser observada uma melhora em praticamente todos os itens observados, concluindo que exercícios em ambiente aquático podem ser benéficos no tratamento da AIJ.

Singh-Grewal et al. ${ }^{23}$, em estudo com 9 crianças com AIJ, buscaram avaliar a segurança e a viabilidade de um programa de exercícios de 3 meses, avaliar a dor durante o exercício, comparar classificaçóes de esforço percebido com frequência cardíaca alcançada e estimar o efeito do treinamento na eficiência metabólica da marcha medida pelo teste de esforço submáximo. Foram utilizados o Childhood Health Assessment Questionnaire (C-HAQ) e Juvenile Arthritis Functional Status, avaliando a qualidade de vida global e saúde relacionada à qualidade de vida. Uma escala visual analógica foi utilizada para avaliar a dor durante os testes e treinamento e a escala de Borg foi utilizada para medir o esforço percebido. O programa de treinamento foi realizado em formato de circuito, envolvendo exercícios na piscina, bicicleta ergométrica, esteira e Fitball. Ao final do programa de treinamento, o custo energético da locomoção foi reduzido na maioria dos indivíduos e o $\mathrm{VO}_{2 \text { pico }}$ aumentou em 4 dos 6 indivíduos, contudo os valores encontrados não foram estatisticamente significativos. A potência anaeróbia de perna melhorou em todos os indivíduos, o pico de potência anaeróbia de braço foi reduzido de forma significativa e a resistência muscular de braço não mostrou alteração. Os indivíduos relataram que as experiências mais agradáveis estavam relacionadas às atividades realizadas na piscina. Levando em consideraçáo os resultados obtidos, pode-se afirmar que crianças com AIJ foram capazes de participar de teste de esforço sem maiores problemas, contudo uma criança com doença grave do quadril abandonou o programa de exercício devido à dor durante as sessóes e piora nos sintomas da artrite. Este estudo sugere que é seguro, viável e aceitável, para crianças com artrite, na ausência de envolvimento grave do quadril, participar de programas de treinamento estruturados.

Van Brussel et al. ${ }^{24}$, em estudo com 62 indivíduos com AIJ, com idade média de 11,9 $\pm 2,2$ anos, buscaram comparar as capacidades aeróbia e anaeróbia desses sujeitos com um grupo controle saudável e determinar se havia diferenças entre subgrupos de indivíduos com AIJ (poliarticular, oligoarticular e sistêmica). Tratou-se de um estudo transversal, sendo concedido tempo de repouso adequado entre os testes. A capacidade aeróbia foi avaliada usando ergoespirometria, e a capacidade anaeróbia usando o Wingate Anaerobic Exercise Test (WAnT); o IMC foi avaliado de acordo com os valores de corte internacionais para crianças com sobrepeso e obesidade, e a adiposidade subcutânea pelas orientaçóes do American College of Sports Medicine (ACSM); avaliaram-se a mobilidade articular de acordo com a pEPM-ROM e a capacidade funcional com a versão holandesa do C-HAQ. Os resultados apresentam uma diminuição significativa na capacidade aeróbia e anaeróbia nas crianças com a AIJ em comparação com o grupo controle; dentro dos subgrupos, pôde-se observar que o grupo oligoarticular não apresentou diferenças significativas em comparação ao grupo controle, já o grupo poliarticular apresentou maior comprometimento aeróbio e anaeróbio, concluindo que crianças com AIJ têm redução significativa das capacidades aeróbias e anaeróbias, principalmente as classificadas como AIJ poliarticular.

Lelieveld et al. ${ }^{25}$, em estudo com 49 jovens, sendo 22 com AIJ (chamado de grupo AIJ) e 27 saudáveis (chamado grupo Wingate), com idade média de 17,1 \pm 0,7, buscaram examinar a capacidade aeróbia e anaeróbia em portadores de AIJ em comparação com indivíduos saudáveis e avaliar a associação entre variáveis da doença e a capacidade para realização desses exercícios. Todos os testes foram realizados no mesmo dia, com o tempo de repouso suficiente entre cada teste. A capacidade anaeróbia foi testada com o WAnT, a capacidade aeróbia com o Symptom Limited Bicycle Ergometry Test (SLBE), a capacidade funcional com uma versão holandesa validada do Childhood Health Assessment Questionnaire (C-HAQ) e a dor e bem-estar geral foram medidos por meio de uma escala visual analógica. Os consumos máximos de oxigênio absoluto e relativo e a potência média de meninas e meninos do grupo AIJ apresentaram resultados significativamente debilitados quando comparados ao grupo Wingate, enquanto a potência de pico teve resultado significativamente debilitado somente para as meninas do grupo AIJ. Já a composição corporal não influenciou substancialmente nos resultados, concluindo que adolescentes com AIJ têm capacidades aeróbia e anaeróbia prejudicadas em relação a seus pares saudáveis pareados por sexo.

Santoni et al. ${ }^{12}$, em estudo com um voluntário com idade de 5 anos, com AIJ classificada como oligoarticular, buscaram avaliar o efeito de uma intervençáo de 
hidroterapia na capacidade funcional e na qualidade de vida. Foram usados métodos quantitativos (ADM, força muscular, flexibilidade e cirtometria de membros inferiores) e métodos qualitativos (anamnese, inspeção, palpação, avaliação da marcha e avaliaçáo postural). Também foi utilizado o questionário SF-36, o qual foi adaptado à faixa etária do paciente e aplicado à sua mãe. A intervenção consistiu em duas sessôes por semana, com uma hora de duraçáo cada, totalizando 10 sessóes, nas quais foram realizadas atividades de aquecimento, alongamentos, exercícios resistidos, exercícios de relaxamento e atividades lúdicas. Foi observada melhora geral na amplitude de movimento articular, na flexibilidade muscular, na capacidade funcional, no estado geral de saúde e na vitalidade, além de redução da dor e cirtometria em membros inferiores, concluindo que a hidroterapia promoveu melhora na capacidade funcional e qualidade de vida nesse indivíduo com AIJ, corroborando com os achados de Degani et al. ${ }^{13}$.

Singh-Grewal et al. ${ }^{26}$ realizaram um estudo controlado, randomizado, cego, com 80 indivíduos com AIJ, idades entre 8 e 16 anos, para examinar a eficácia do treinamento aeróbio de alta intensidade em comparação com o treinamento de baixa intensidade, em termos de custo energético da locomoçáo, captação de oxigênio de pico, potência de pico e função física autorrelatados. O grupo controle foi composto por 39 indivíduos e o grupo experimental, por 41 indivíduos. Os indivíduos participaram de um programa de formação durante 12 semanas, com 3 sessóes por semana, consistindo em exercício aeróbio de alta intensidade no grupo experimental e qigong (que consiste de exercícios suaves, com movimentos repetitivos de alongamento, com intuito de aumentar a circulação e desenvolver uma melhor consciência corporal) no grupo de controle. Os indivíduos foram submetidos a teste de esforço para medir o consumo de oxigênio submáximo a $3 \mathrm{~km} / \mathrm{h}\left(\mathrm{VO}_{\text {2submax }}\right)$, consumo máximo de oxigênio $\left(\mathrm{VO}_{2 \max }\right)$ e potência de pico no início e no final do programa. A funçáo física foi medida usando o C-HAQ. Náo houve diferença significativa ao final do treinamento, todavia os resultados sugerem que programas de atividade com ou sem um componente de treinamento aeróbio são seguros e podem resultar em uma importante melhora na funçáo física. Além disso, foi evidenciada maior adesão ao programa qigong, podendo sugerir que regimes menos intensivos são mais fáceis para crianças com AIJ e proporcionam um grau de benefício equivalente ao dos programas intensivos.

Tarakci et al. ${ }^{27}$, em um estudo controlado, randomizado, cego, com 81 indivíduos com AIJ, com idades entre 5 e 17 anos, buscaram investigar os efeitos de um programa de treinamento com exercícios no solo, para avaliar a dor, capacidade funcional e qualidade de vida. Os pacientes foram aleatoriamente divididos em grupo de intervenção $(n=43)$ e grupo controle $(n=38)$. As variáveis foram avaliadas por meio do teste de caminhada de 6 minutos, do C-HAQ, de uma escala visual analógica e do Pediatric Quality of Life Inventory Module Arthritis (PedsQL). O grupo intervenção realizou 4 sessões de exercício por semana, durante 12 semanas, e os programas de exercícios eram planejados individualmente por um fisioterapeuta. O treinamento consistia em exercícios para mobilidade articular, de fortalecimento muscular, alongamentos e exercícios posturais, incentivando a utilizaçáo mais frequente da extremidade afetada. Ao final do período de treinamento, foi notada uma melhora em todas as avaliaçóes no grupo exercício, concluindo que o programa de exercícios de solo pode resultar na melhoria da função física e qualidade de vida em indivíduos com AIJ.

\section{Discussão}

Considerando os principais desfechos do treinamento físico na AIJ, pode-se afirmar que:

1) dos três estudos que utilizaram de exercícios no meio líquido para avaliar a qualidade de vida e capacidade funcional de indivíduos portadores de AIJ, tanto Degani et al. ${ }^{13}$ quanto Santoni et al. ${ }^{12}$ evidenciaram melhoras significativas na amplitude de movimento articular, qualidade de vida relacionada a saúde e reduçáo da dor. Degani et al. ${ }^{13}$ encontraram, ainda, redução da utilização de órteses com o uso da intervenção aplicada. No estudo conduzido por Singh-Grewal et al. ${ }^{23}$, foi encontrada evidência de aumento da potência anaeróbia de perna. Isto posto, pode-se afirmar que treinamento físico no meio líquido propicia benefícios a indivíduos com AIJ;

2) com relação aos estudos que utilizaram intervenção por meio de exercícios aeróbios, Lelieveld et al..$^{25} \mathrm{e}$ Van Brussel et al. ${ }^{24}$ chamaram atenção para o fato de que indivíduos com AIJ apresentaram menor capacidade aeróbia que seus pares saudáveis. Contudo, ao avaliarem o efeito desse tipo de intervenção, Singh-Grewal et al. ${ }^{26}$ observaram melhora nas funçôes físicas de uma forma geral, e Singh-Grewal et al. ${ }^{23}$ evidenciaram reduçấo do custo energético de locomoção e aumento do $\mathrm{VO}_{2 \text { pico, }}$ o que sugere que o treinamento aeróbio é importante para a promoção da capacidade aeróbia de indivíduos com AIJ; 
3) Santoni et al. ${ }^{12}$, Degani et al. ${ }^{13}$ e Tarakci et al. ${ }^{27}$ propuseram a realização de exercícios para mobilização articular e alongamentos, os quais demonstraram melhora na ADM e na QVRS, com especial destaque para o estudo de Tarakci et al. ${ }^{27}$, que apresentou, ainda, melhora na capacidade funcional, no fortalecimento muscular, na flexibilidade, na postura e redução da dor.

Em síntese, na maioria dos estudos, independentemente do tipo de treinamento e de acordo com o que é corroborado pelas recomendaçóes do American College of Rheumatology ${ }^{17}$, tanto o treinamento aeróbio, o treinamento resistido, os exercícios de flexibilidade, os alongamentos e/ou os exercícios de equilíbrio, quer sejam em meio líquido ou no solo, são os tipos de atividades recomendadas para pessoas com AIJ e, além de trazerem inúmeros benefícios, podem ser complementares a terapia medicamentosa.

\section{Conclusões}

Embora o conjunto dos fatores que contribuem para a ocorrência da AR seja extensamente estudado, principalmente fatores genéticos e imunológicos, os mecanismos precisos envolvidos na iniciação, progressão e destruição autoimune presentes especificamente na AIJ náo estão totalmente elucidados.

Enquanto os medicamentos podem diminuir a dor e a inflamação, o treinamento físico, quer seja no solo ou em meio líquido, pode auxiliar a manter e melhorar a mobilidade articular, evitando a perda da capacidade elástica dos tecidos, mantendo a flexibilidade do sistema musculoesquelético e prevenindo deformidades, encurtamentos musculares e limitaçóes das articulaçôes. Fortalecer a musculatura auxilia, ainda, nas atividades de vida diárias, tais como: andar, comer, escrever, brincar, entre outras. Contudo, aconselha-se que antes do início de qualquer a atividade física, o indivíduo com AIJ seja encaminhado para avaliação e acompanhamento de uma equipe multidisciplinar.

As evidências explicitadas sugerem que o treinamento físico pode e deve ser estimulado e incorporado à terapia medicamentosa para beneficiar indivíduos com AIJ, atenuando sintomas clínicos relacionados à doença.

Constata-se, contudo, a necessidade de aprofundar os estudos sobre os efeitos do treinamento físico na AIJ, pois há poucas pesquisas relatadas, o que dificulta o estabelecimento de diretrizes sobre a prescrição de exercício para essa população. Assim, existe uma urgente necessidade de maiores estudos randomizados a fim de examinar questóes referentes à prática de atividade física e exercício físico por indivíduos vivendo com AIJ.

\section{Referências}

1. Bielemann RM, Silva BGC, Coll CVN, Xavier MO, Silva SG. Burden of physical inactivity and hospitalization costs due to chronic diseases. Rev Saúde Pública. 2015;49:75.

2. Santos AL, Garcia Junior JR. Atividade física e dieta como meios preventivos da obesidade infantil. RBONE. 2012;6(31):23-30.

3. Gualano B, Pinto ALS, Perondi MB, Roschel H, Sallum AME, Hayashi APT, et al. Efeitos terapêuticos do treinamento físico em pacientes com doenças reumatologias pediátricas. Rev Bras Reumatol. 2011;51(5):484-96.

4. Faria JL. Patologia geral: fundamentos das doenças com aplicaçóes clínicas. Rio de Janeiro: Guanabara Koogan; 2003.

5. National Institute of Arthritis and Musculoskeletal and Skin Diseases. Understanding autoinflammatory diseases. 2016 [citado em 2016 jul 6]. Disponível em: https:/goo.gl/

6. American College of Rheumatology. Autoimmune diseases. Atlanta (GA): ACR; c2016 [citado em 2016 jul 6]. Disponível em: https://goo.gl/ssntB5

7. Chiarello B, Driusso P, Radl ALM. Fisioterapia reumatológica. Barueri: Manole; 2005.

8. American College of Rheumatology. Rheumatoid Arthritis. Atlanta (GA): ACR; c2016 [citado em 2016 jul 6]. Disponível em: https://goo.gl/Of9ZrN

9. Laurindo IMM, Ximenes AC, Lima FAC, Pinheiro GRC, Batistella LR, Bertolo MB, et al. Artrite reumatóide: diagnóstico e tratamento. Rev Bras Reumatol. 2004;44(6):435-42.

10. Sociedade Brasileira de Reumatologia. Artrite Reumatoide. São Paulo: SBR; c2016 [citado em 2016 jul 6]. Disponível em: https://goo.gl/WwN45e

11. Ringold S, Weiss PF, Beukelman T, DeWitt EM, Ilowite NT, Kimura Y, et al. 2013 update of the 2011 American College of Rheumatology recommendations for the treatment of juvenile idiopathic arthritis: recommendations for the medical therapy of children with systemic juvenile idiopathic arthritis and tuberculosis screening among children receiving biologic medications. Arthritis Rheum. 2013;65(10):2499-512.

12. Santoni FC, Freitas SCP, Oliveira J, Mesquita RA. Hidroterapia e qualidade de vida de um portador de artrite reumatoide juvenil - estudo de caso. Fisioter Mov. 2007;20(1):101-8.

13. Degani AM, Villa PS. Amplitude de movimento articular e qualidade de vida relacionada à saúde de portador de artrite idiopática juvenil submetido à fisioterapia aquática. Fisioter Mov. 2005;18(4):33-42. 
14. Kumar V, Abbas AK, Fausto N. Robbins \& Cotran Patologia - Bases patológicas das doenças. Rio de Janeiro: Elsevier; 2005.

15. Sakamoto AP, Peracchi OAB, Terreri MTRA, Len CA. Artrite idiopática juvenil. Pediatr Mod. 2013;49(9):398-402.

16. Wibelinger LM. Fisioterapia em reumatologia. Rio de Janeiro: Revinter; 2009.

17. American College of Rheumatology. Artritis juvenil. Atlanta (GA): ACR; c2015 [citado em 2016 jul 6]. Disponível em: https://goo.gl/QMw7LG

18. Beukelman T, Patkar NM, Saag KG, Tolleson-Rinehart S, Cron RQ, DeWitt EM, et al. 2011 American College of Rheumatology recommendations for the treatment of juvenile idiopathic arthritis: initiation and safety monitoring of therapeutic agents for the treatment of arthritis and systemic features. Arthritis Care Res (Hoboken). 2011;63(4):465-82.

19. Boletim Brasileiro de Avaliação de Tecnologias em Saúde. Medicamentos biológicos para o tratamento da artrite reumatoide. 2012;VI(19). [citado em 2016 maio 6]. Disponível em: https://goo.gl/ChnFLo

20. Roschel H, Tricoli V, Ugrinowitsch C. Treinamento físico: consideraçóes práticas e científicas. Rev Bras Educ Fís Esp. 2011;25(Esp):53-65.

21. Gualano B, Pinto ALS, Perondi B, Prado DML, Omori CA, Almeida RT, et al. Evidence for prescribing exercise as treatment in pediatric rheumatic diseases. Autoimmun Rev. 2010;9(8):569-73.

22. Klepper SE. Exercise in pediatric rheumatic diseases. Curr Opin Rheumatol. 2008;20(5):619-24.

23. Singh-Grewal D, Wright V, Bar-Or O, Feldmann BM. Pilot study of fitness training and exercise testing in polyarticular childhood arthritis. Arthritis Rheum. 2006;55(3):364-72.

24. van Brussel M, Lelieveld OTHM, van der Net J, Engelbert RHH, Helders PJM, Takken T. Aerobic and anaerobic exercise capacity in children with juvenile idiopathic arthritis. Arthritis Rheum. 2007;57(6):891-7.

25. Lelieveld OTHM, van Brussel M, Takken T, van Weert E, van Leeuwen MA, Armbrust W. Aerobic and anaerobic exercise capacity in adolescents with juvenile idiopathic arthritis. Arthritis Rheum. 2007;57(6):898-904.

26. Singh-Grewal D, Schneiderman-Walker J, Wright V, BarOr O, Beyene J, Selvadurai $\mathrm{H}$, et al. The effects of vigorous exercise training on physical function in children with arthritis: a randomized, controlled, single-blinded trial. Arthritis Rheum. 2007;57(7):1202-10.

27. Tarakci E, Yeldan I, Baydogan SN, Olgar S, Kasapcopur O. Efficacy of a land-based home exercise programme for patients with juvenile idiopathic arthritis: a randomized, controlled, single-blind study. J Rehabil Med. 2012;44(11):962-7.

\section{Como citar este artigo:}

Silveira JS, Moura VW, Toigo AM. Efeitos do exercício físico no tratamento da artrite idiopática juvenil: uma revisão sistemática. Rev. Aten. Saúde. 2017;15(54):90-99. 